\title{
DOES FASTING AND POSTPRANDIAL BLOOD SAMPLING FOR THYROID EVALUATION MAKE A DIFFERENCE IN CHILDREN?
}

\author{
Poovazhagi Varadarajan¹, Arivoli Kaliyan², Suresh Kumar Sankaran³, Anupama Sankaran4, Nisha Rangabhashyam ${ }^{5}$, \\ Asvatha Chinnarajalu6 \\ 1 Professor, Department of Paediatrics, Chengalpattu Medical College, Chengalpattu. \\ ${ }^{2}$ Assistant Professor, Department of Paediatrics, Chengalpattu Medical College, Chengalpattu. \\ ${ }_{3}^{3}$ Assistant Professor, Department of Paediatrics, Chengalpattu Medical College, Chengalpattu. \\ ${ }^{4}$ Resident, Department of Paediatrics, Chengalpattu Medical College, Chengalpattu. \\ ${ }^{5}$ Resident, Department of Paediatrics, Chengalpattu Medical College, Chengalpattu. \\ ${ }^{6}$ Resident, Department of Paediatrics, Chengalpattu Medical College, Chengalpattu.
}

ABSTRACT

\section{BACKGROUND}

Thyroid function tests are one of the commonly undertaken investigations in any paediatric outpatient department. Based on the existing literature, there are no standard guidelines in respect of the sampling procedure to be followed in infants and children. Every paediatrician is aware of the logistic problems in taking a fasting sample in infants and younger children unlike adults. In this context, it was planned to undertake a pilot study to identify if the timing of sampling, fasting or postprandial status, makes any difference with respect to the thyroid function tests in children

\section{MATERIALS AND METHODS}

This was a descriptive study undertaken at the paediatric unit of Chengalpattu Medical College to evaluate if there is any difference between fasting and postprandial blood levels of Thyroid stimulating hormone and free T4 in children. The study was approved by the institutional ethical committee and informed written consent and assent obtained prior to study. Fifty one children between 1 and 12 years of age were recruited based on their willingness, from the endocrine clinic and the paediatric outpatient clinic Fasting and postprandial blood samples were collected by venepuncture at 8 a.m. and 10.15 a.m. respectively. Blood samples were analysed using electrochemiluminescence method. Data analysis was done using Epi info software.

\section{RESULTS}

51 children underwent fasting and postprandial sampling for thyroid evaluation. The results did not reveal any statistically significant difference between the fasting and postprandial samples for both TSH and Free T4. The same was true for both hypothyroid and euthyroid children. Subclinical hypothyroidism was encountered in one child in the fasting sample.

\section{CONCLUSION}

This pilot study revealed that postprandial TSH and Free T4 can be used as reliable test for thyroid evaluation as is a fasting sample in children.

\section{KEYWORDS}

Thyroid Function Test, Fasting Sample, Postprandial Sampling.

HOW TO CITE THIS ARTICLE: Varadarajan P, Kaliyan A, Sankaran SK, et al. Does fasting and postprandial blood sampling for thyroid evaluation make a difference in children?. J. Evolution Med. Dent. Sci. 2017;6(19):1515-1518, DOI: $10.14260 /$ Jemds/2017/333

\section{BACKGROUND}

Hypothyroidism is one of the common endocrine problems encountered in children. Reported incidence of hypothyroidism in children is $0.135 \%$.(1) Hospital based studies from western India have documented $93.8 \%$ of thyroid dysfunction among children to be hypothyroidism.(2) A Mumbai based study among 800 children identified hypothyroidism in 79\%.(3) TSH and Free T4 are considered to be the screening tests for thyroid dysfunction.(4) Not all children present with clinical features of hypothyroidism. Subclinical hypothyroid states need to be identified as well.

Financial or Other, Competing Interest: None.

Submission 12-12-2016, Peer Review 21-02-2017,

Acceptance 27-02-2017, Published 06-03-2017.

Corresponding Author:

Dr. Poovazhagi Varadarajan,

Professor, Department of Paediatrics,

Chengalpattu Medical College, Chengalpattu.

E-mail: poomuthu@gmail.com

DOI: $10.14260 /$ jemds $/ 2017 / 333$

\section{(c) $($ ) $९$}

Thyroid function tests are one of the common investigations in paediatric outpatient departments. In adults and older children, fasting sampling can be done. However, fasting samples in infants and younger children needs overnight fasting for 6 hours and early morning blood sampling. Literature search conducted to know if thyroid testing warrants fasting sample did not reveal any guidelines. Hence, this study was undertaken to look for any significant difference in fasting and postprandial values of TSH and Free $\mathrm{T} 4$ in the blood samples from children. If postprandial values are significantly different, it then becomes mandatory that fasting samples are collected for thyroid evaluation.

Justification- Thyroid function test is one of the common investigation in paediatric outpatient department. Based on the existing literature, there are no standard guidelines for blood sampling in infants and children. In adults, thyroid function tests are being done as a part of investigations for hypertension, hyperlipidaemia and hyperglycaemia, hence is taken as a fasting sample. Every paediatrician is aware of the difficulty in taking a 6- to 8-hour fasting sample in infants and 
younger children. In this context, it was planned to undertake a pilot study to identify if the timing of sampling, fasting or postprandial status, makes any difference in the thyroid function test results in children.

The aim was to compare the levels of thyroid stimulating hormone (TSH) and free T4 in fasting and postprandial state among children.

\section{MATERIAL AND METHODS}

This is a hospital based prospective observational study.

Setting- This study was undertaken at the paediatric outpatient department of a semiurban paediatric referral centre at Chengalpattu under the Government of Tamil Nadu.

Study Duration - Between Sep. 2016 and Oct. 2016.

Sample Size- 51 children- as it was a pilot study.

\section{Sampling Technique}

Children attending the paediatric outpatient clinic for followup after minor illness and the endocrine clinic of the department with already diagnosed hypothyroidism were consecutively selected for the study based on their willingness to participate for sampling. Children and the parents/caregivers were informed of the nature of the study.

\section{Study Parameters}

Age, gender, nature of the sample fasting or postprandial for TSH and Free T4 estimation.

\section{Exclusion Criteria}

Infants were excluded since fasting sample could not be undertaken as outpatients.

\section{Manoeuvre}

Children were recruited following informed written consent from the parents or the caregivers and assent if applicable. They were asked to come to the OPD on the next day morning with fasting of at least 6 hours. Fasting blood samples were collected at 8 a.m. and 2-hour postprandial samples were collected at $10.15 \mathrm{am} .2 \mathrm{~mL}$ venous blood was collected following standard venepuncture on both the occasions and the samples were analysed in the laboratory for thyroid stimulating hormone and free T4 levels. The samples were analysed using electrochemiluminescence assay.

\section{Statistical Methods}

Data was analysed using EPI Info version 7.0. Mean and standard deviation were calculated for the Thyroid stimulating hormone and Free T4. Differences in free T4 and TSH levels between fasting and postprandial states were analysed using paired student $t$ test and $p$ values below 0.05 was considered statistically significant.

The study was undertaken after institutional ethics approval and informed written consent or assent as applicable.

\section{RESULTS}

51 children were recruited to undergo both fasting and postprandial serum sampling for Free T4 and TSH. 27 girls and 24 boys were evaluated. Gender distribution revealed a male female ratio of 1.12:1. Among the study group, 28 children were under 5 years of age, 13 children were between 6-10 years and 10 children were above 10 years. Among the 51 children, 40 children were euthyroid and 11 children were hypothyroid.

The fasting T4 values ranged from 0.58 to $1.93 \mathrm{ng} / \mathrm{dL}$ with a mean (SD) of $1.22( \pm 0.265) \mathrm{ng} / \mathrm{dL}$. The postprandial values ranged from 0.52 to $1.72 \mathrm{ng} / \mathrm{dL}$ with a mean of 1.201 $\pm 0.245) \mathrm{ng} / \mathrm{dL}$. Paired t- test analysis revealed the P 0.112 for fasting samples.

Fasting values of TSH ranged from 0.23 to $150 \mathrm{mIU} / \mathrm{L}$ with a mean of $11.36( \pm 35.09) \mathrm{mIU} / \mathrm{L}$. Postprandial TSH values ranged from 0.24 to $150 \mathrm{mIU} / \mathrm{L}$ and a mean of 11.32 ( \pm 35.11) $\mathrm{mIU} / \mathrm{L}$. Paired t test revealed $P$ to be 0.682 .

Comparison of means of fasting and postprandial Free T4 and TSH did not reveal any statistically significant difference with a P-0.095 and 0.4134 respectively.

Among the euthyroid group, Free T4 values paired t test revealed the following results in fasting. Values from 0.800 to $1.93 \mathrm{ng} / \mathrm{dL}$ with a mean of 1.28 95\% CI 1.206- 1.357 (0.237) ng/dL. Among the postprandial T4 ranged from 0.790 to 1.72 $\mathrm{ng} / \mathrm{dL}$ with a mean of $1.2695 \%$ CI $1.189-1.325$ (0.212) ng/dL. The arrived $P$ value was 0.133 not significant.

Among the hypothyroid group, Free T4 analysis revealed the fasting values ranged from $0.58-1.39 \mathrm{ng} / \mathrm{dL}$ with a mean of $1.0195 \%$ CI $0.831-1.182(0.261) \mathrm{ng} / \mathrm{dL}$ and among the postprandial group in free T4 values from $0.520-1.38 \mathrm{ng} / \mathrm{dL}$ and mean of $0.99895 \%$ CI $0.826-1.17(0.256) \mathrm{ng} / \mathrm{dL}$. The paired t test $p$ value was 0.621 among the hypothyroid group.

Among the euthyroid group, TSH values revealed the following in paired $t$ test. Fasting values from 0.23-5.46 $\mathrm{mIU} / \mathrm{L}$ with a mean of $2.3395 \%$ CI 1.986-2.7712(1.37) mIU/L. The postprandial value ranging from $0.24-4.54 \mathrm{mIU} / \mathrm{L}$ with a mean of $2.2195 \%$ CI 1.19-2.591(1.21) mIU/L. The paired $t$ test $p$ value was 0.321 .

Among the hypothyroid group, fasting TSH values ranged from $0.34-150 \mathrm{mIU} / \mathrm{L}$ with a mean of $44.295 \%$ CI- 1.53-89.97 (68.1) $\mathrm{mIU} / \mathrm{L}$, and among the PP group ranged from 0.400 to 150 with a mean of $44.595 \%$ CI $-1.163-90.13(67.9) \mathrm{mIU} / \mathrm{L}$. The paired t test $\mathrm{p}$ value was 0.058 and was not significant.

\section{DISCUSSION}

Thyroid function tests are one of the common tests done for endocrine evaluation in children. It is well known that TSH values fluctuate throughout the day, and recent studies have demonstrated that TSH is significantly reduced in the fed state, with no significant variation in $\mathrm{T} 4$ levels between the fasted and fed states.(5) TSH and Free T4 are the best markers of thyroid function assessment. Elevated TSH and lower FT4 indicate hypothyroidism. If the TSH level is slightly raised but the FT4 level is still within the normal reference range, this is called subclinical hypothyroidism or mild thyroid failure.(6) In the absence of robust literature evidence of need for fasting samples for thyroid function evaluation in children, there is a need for data on fasting and postprandial samples. This pilot study has shown no significant difference between the fasting and postprandial values of TSH and Free T4 in children. The concern raised in favour of fasting samples is to pick up subclinical hypothyroidism which is of a major concern in 
certain risk groups. Existing literature favours thyroxin supplementation among adults and certain risk groups like pregnancy; however, this may not be applicable to children.

Subclinical hypothyroidism in children rarely develops into overt or clinical hypothyroidism; an additional test for thyroid antibodies will help to determine the risk. Some people with subclinical hypothyroidism, particularly those whose TSH level is greater than $10 \mathrm{mIU} / \mathrm{L}$, may benefit from treatment with levothyroxine.(7) The outcome of subclinical hypothyroidism in children is a matter of debate.(8) A low TSH with a low FT4 may be a result of a failure of the pituitary gland (Secondary hypothyroidism caused by hypopituitarism) or a response to a significant non-thyroid illness. TSH shows diurnal variation with peak level between 11.00 p.m. - 5.00 a.m. due to circadian rhythm and lowest between 5.00 a.m. - 8.00 p.m.(7) The pulsatile secretion of TSH does not produce much circulatory variations because of low amplitude TSH secretion and long half-life of TSH. Pulses of TSH secretion has been documented between 20.00 hours and 04.00 hours.(9) A study conducted among 57 adult patients by Mahadevan S et al(10) concluded that fasting state does influence thyroid function test results especially TSH values. Not much changes were documented in Free T4 values. This might affect the diagnosis of subclinical hypothyroid disease in the study population. However, the study population is not comparable as it involves children in the present study. It is observed generally that TSH in the fasting state was higher than the levels measured later in the same day. But there was no documented statistical significance between fasting and postprandial TSH values. Similar studies from elsewhere have documented that fasting and postprandial samples do not yield different results provided they are taken in the morning.(11) There is no similar literature available in children for thyroid function test to be done only in fasting state. Subclinical hypothyroidism was not a significant problem in this study. Only one child had a TSH value suggestive of subclinical hypothyroidism in the fasting TSH value. Based on the existing evidence the mild forms of untreated subclinical hypothyroidism do not seem to be associated with impairments in growth, bone health or neurocognitive outcome. $(6,12)$ Currently, no studies recommend treatment for these children. However, a small subset with increased antibodies, female gender, family history of thyroid disorder or goitre need to be followed up for development of overt hypothyroidism.(7,13) Spontaneous reversibility of elevated TSH was found in $41-73 \%$ of subjects.(14,15) Mild longstanding subclinical hypothyroidism may be associated with subtle proatherogenic abnormalities in children and may need followup.(16) Since subclinical hypothyroidism is not a significant medical illness with need for thyroxin therapy in children, both fasting sample and postprandial samples can be considered to be useful for evaluation of children with suspected thyroid dysfunction. In this study group, the fasting and postprandial values did not vary significantly for both TSH and Free T4. The findings have been the same for both euthyroid and hypothyroid children.

\section{CONCLUSION}

There is no statistically significant difference between the means of fasting and postprandial values of Free T4 and TSH among children. There was no statistically significant difference in the paired $t$ test between the fasting and postprandial values of both Free T4 and TSH in children with hypothyroid and euthyroid state. Thyroid function testing (Free T4 and TSH) in children may not require a fasting state and can be undertaken irrespective of the feeding status.

\section{Limitations}

Smaller sample size is the major limitation of this study. Influence of the type of food taken and the timing of the sample 8 a.m. and 10.15 a.m. on the values of TSH could not be ascertained as the TSH flow is pulsatile with variation. The role of dietary change in altering the TSH and Free T4 levels need to be addressed as well.

\section{REFERENCES}

[1] Hunter I, Greene S, MacDonald T, et al. Prevalence and aetiology of hypothyroidism in the young. Archives of Disease in Childhood 2000;83(3):207-10.

[2] Singh A, Purani C, Mandal A, et al. Prevalence of thyroid disorders in children at a tertiary care hospital in western India. Journal of Clinical and Diagnostic Research 2016;10(2):SC01-4.

[3] Unnikrishnan AG, Menon UV. Thyroid disorders in India: an epidemiological perspective. Indian J Endocr Metab 2011;15(Suppl 2):S78-81.

[4] Carvalho GA, Perez CL, Ward LS. The clinical use of thyroid function tests. Arquivos Brasileiros de Endocrinologia \& Metabologia 2013;57(3):193-204.

[5] McGregor, Brock. Extra-Thyroidal factors impacting thyroid hormone homeostasis: a review. Journal of Restorative Medicine 2015;4(1):40-9.

[6] Surks MI, Sievert R. Drugs and thyroid function. N Engl J Med 1995;333(25):1688-94.

[7] Shriram M, Sridhar M. Subclinical hypothyroidism in children. Indian Pediatr 2014;51(11):889-95.

[8] Salerno M, Capalbo D, Cerbone M, et al. Subclinical hypothyroidism in childhood-current knowledge and open issues. Nat Rev Endocrinol 2016;12(12):734-46.

[9] Brabant G, Prank K, Ranft U, et al. Physiological regulation of circadian and pulsatile thyrotropin secretion in normal man and woman. The Journal of Clinical Endocrinology \& Metabolism 1990;70(2):4039.

[10] Nair R, Mahadevan S, Muralidharan RS, et al. Does fasting or postprandial state affect thyroid function testing? Indian J Endocrinol Metab 2014;18(5):705-7.

[11] Niranjan U, Wright NP. Should we treat subclinical hypothyroidism in obese children? BMJ 2016;352:i941.

[12] Bandophadhyay D, Goel P, Baruah H, et al. Fasting or random: which venous sample is better for thyroid function testing. Journal of Advanced Research in Biological Sciences 2012;4(4):275-8.

[13] Kaplowitz PB. Subclinical hypothyroidism in children: normal variation or sign of a failing thyroid gland? Int J Pediatr Endocrinol 2010;2010:281453. 
[14] Lazar L, Frumkin RBD, Battat E, et al. Natural history of thyroid function tests over 5 years in a large pediatric cohort. Journal of Clinical Endocrinology and Metabolism 2009;94(5):1678-82.

[15] Wasniewska M, Salerno M, Cassio A, et al. Prospective evaluation of the natural course of idiopathic subclinical hypothyroidism in childhood and adolescence. European Journal of Endocrinology 2009;160(3):471-21.
[16] Cerbone M, Capalbo D, Wasniewska $M$, et al. Cardiovascular risk factors in children with longstanding untreated idiopathic subclinical hypothyroidism. J Clin Endocrinol Metab 2014;99(8):2697-703. 